Annals of Pure and Applied Mathematics

Vol. 21, No. 1, 2020, 55-61

ISSN: 2279-087X (P), 2279-0888(online)

Published on 14 March 2020

www.researchmathsci.org

DOI: http://dx.doi.org/10.22457/apam.v21n1a7659

Annals of

Pure and Applied

Mathematics

\title{
On the Holographic Principle Description for the Viscous Incompressible Fluids
}

\author{
Javier Rivera \\ 865 River Boat Circle, Orlando, Florida 32828 \\ E-mail: caribe31415@gmail.com \\ (C) 2019 USA Registration \# TXu-2-179-567
}

Received 14 February 2020; accepted 11 March 2020

\begin{abstract}
The field derivative of the incompressible fluid's Bernoulli equation is shown to be mathematically equivalent to the addition of the dot product of the fluid velocity with the Navier-Stokes equation added to the Laplace equation of the classical Lagrangian of the incompressible viscous fluid per unit mass specified for every time, $t$, within a given finite time interval within a control volume. Since the Navier-Stokes equations are zero, this implies the Laplacian operating on the fluid's classical Lagrangian per unit mass is also zero. Solving the Laplace equation of the fluid's classical Lagrangian for a given time $t$ for a given set of boundary condition will produce a Lagrangian scalar field, which it may be potentially could be solved for values of Eulerian fluid velocity and pressure through the control volume domain and could be used to produce a holographic movie of Eulerian velocity flow field and potential field within the control volume domain.
\end{abstract}

Keywords: Bernoulli Equation, Navier-Stokes equation, Laplace equation, Lagrangian, Holographic Principle

AMS Mathematics Subject Classification (2010): 76D05, 35Q35

\section{Introduction}

The Laplace equation of the fluid velocity potential has been well known to be used in inviscid incompressible fluid flows in the literature [5,6]. This article will show the use of the Laplace equation is not limited to inviscid incompressible fluid flows, but the Laplace equation can be also used for incompressible viscous flows with the interpretation Holographic Principle. Reference [7] shows the Holographic Principle has been used in physics to develop understanding on Turbulence Kolmogorov scaling and its relation to quantum foam and quantum gravity. Reference [8] provides description on how the holographic principle has been used to understand quantum physics as "if the visible universe were reading of a lower dimensional hologram generated in hyperspace". Reference [9] reviews developments in holographic dynamics with the aim to understand different techniques of transport coefficients computation with the use of string theory. Reference [10] actually applies the methods of holography in Fluid Mechanics and particle dynamics with the use of strong optical signal processing. 


\section{Javier Rivera}

The thesis of this article has a different perspective as compared to different references above [7-10]. In this article for each fixed time, the holographic principle corresponds to the boundary conditions within the fixed arbitrary Eulerian control volume domain, $W_{o}$, that are considered to represent the lower dimensional "hologram which is projected via the Laplace equation of the classical fluid's Lagrangian per unit mass by propagating the boundary conditions to the Eulerian control volume domain, $W_{o}$.

The article [1]entitled "On the Field Derivatives and its Application to Fluids" derived the Bernoulli equation for incompressible fluid which is a specified constant along a streamline for sampled times, $t_{k}$, within a given arbitrary finite time interval $[0, t 1]$. The innovation of [1] was to derive the field derivative concept and the field derivative application to the Bernoulli equation yields zero since the Navier-Stokes equation is obtained. Unlike reference [1] or reference [3], in this article all spatial and velocity components are Eulerian coordinates and control volumes are fixed or/and arbitrary. The Einstein summation convention is used throughout this manuscript with indices $\mathrm{j}$ and $\mathrm{i}$ equal to 1,2 and 3 , unless otherwise noted.

The application of the field derivative to the incompressible viscous fluid's Bernoulli equation results in a time static Laplace equation of the classical fluid Lagrangian per unit mass, which is independent of viscous effects, since the NavierStokes equation holds throughout the volume domain. Therefore, the dot product of the fluid's velocity with the Navier-Stokes component equation can be interpreted to be equivalent to a time static Laplace equation of the classical Lagrangian per unit mass, which is independent of viscous effect. Since the Laplace equation of the Lagrangian is linear and therefore superposition of solutions is allowed.

Solving the Laplace equation of the classical Lagrangian per unit mass for given sample times, $t_{k}$, and given set of boundary conditions will produce a solution set of volumetric scalar fields of the Lagrangian per unit mass in Eulerian fixed space dependent on the boundary conditions for each sample time. The solution volumetric scalar field of the Lagrangian may be potentially solved for values of Eulerian fluid velocity and pressure through the volume domain similarly or analogously to a holographic movie of the fluid flow, although numerical methods may need to be used to track the Lagrangian fluid flow. This Eulerian fluid volumetric flow dependence on surface boundaries conditions at a given instant of time via the Laplace equation of the classical Lagrangian is similar or analogous to the "holographic principle" described in Roger Penrose's The Road to Reality book in page 920 [2].

\section{The Eulerian Bernoulli Equation for incompressible viscous fluids}

The Eulerian Bernoulli equation for incompressible viscous fluid was specified in reference [1] as seen in (Eq. 1)which is a constant, $B_{o}$, along the streamline for every time $t$ within a given time interval $[0, t 1]$.

$$
B_{o}\left(t, \vec{x}(t), \vec{u}(t, \vec{x}(t))=\frac{1}{2} u_{i}^{2}(t, \vec{x}(t))+\frac{p(\vec{x}(t))}{\rho_{o}}+\phi(\vec{x}(t))+\int_{0}^{t} \frac{1}{\rho_{o}} \tau_{i, j} \frac{\partial u_{i}}{\partial x_{j}} d s+I_{o}=B_{o}\right.
$$

The innovation of reference [1] was to derive the field derivative concept and showing the field derivative applied to the Bernoulli equation $(E q$. 1) after volume integration by parts within a time independent arbitrary control volume, $W_{o}$, with the usual no slip for the fixed boundary conditions or/and zero shear stress for arbitrary control volume, yields 
On the Holographic Principle Description of the Viscous Incompressible Fluids

zero since the scalar or dot product of the velocity components and Navier-Stokes equation components $(E q .2)^{l}$ are obtained.

$$
\iiint_{W_{o}} \frac{d B_{o}}{d t} \rho_{o} d W=\iiint_{W_{o}} u_{i}\left(\frac{\partial u_{i}}{\partial t}+u_{j} \frac{\partial u_{i}}{\partial x_{j}}+\frac{\partial \phi}{\partial x_{i}}+\frac{1}{\rho_{o}} \frac{\partial p}{\partial x_{i}}-\frac{1}{\rho_{o}} \frac{\partial \tau_{i, j}}{\partial x_{j}}\right) \rho_{o} d W=0
$$

Since the no slip conditions for solid boundaries imply zero velocity field or/and the fluid shear, $\tau_{i, j}$, is zero in absence of a solid boundaries since the surface normal of the control volume boundary may be arbitrary and time independent; therefore, the surface integral due to volume integration by parts is identically zero,

$$
\left[\oiint_{\partial W_{o}} \int_{0}^{t} \frac{1}{\rho_{o}} \tau_{i, j} u_{i} d s \rho_{o} n_{j} d S\right]_{u_{i}=0 \text { or } / \text { and } \tau_{i, j}=0 \text { at } \partial W_{o}}=0 \text {. }
$$

Thus, field or material differentiation with respect to time of the surface integral is still zero since the right side of the equation being differentiated is a constant zero [1] as shown in (Eq. 2A).

$$
\frac{d}{d t} \oiint_{\partial W_{o}} \int_{0}^{t} \tau_{i, j} u_{i} d s n_{j} d S=\oiint_{\partial W_{o}} \tau_{i, j} u_{i} n_{j} d S=0
$$

Therefore, the field derivative of the Bernoulli equation is zero and the Bernoulli equation is constant along the streamline. This is a restatement of what was found in reference [1]. What was missed in reference [1] is that this surface integral can be converted to the volume integral of the Laplacian operating on the fluid's classical Lagrangian per unit mass via the Gauss divergence theorem (see Appendix A). (Eq.1).

By substituting the fluid shear stress for incompressible viscous fluids given in

$$
\tau_{i, j}(s, \vec{x}(s))=2 \mu e_{i, j}(s, \vec{x}(s))=\mu\left(\frac{\partial u_{i}(s, \vec{x}(s))}{\partial x_{j}}+\frac{\partial u_{j}(s, \vec{x}(s))}{\partial x_{i}}\right)
$$

The Bernoulli equation( $E q .1)$ can be restated as $(E q .3)$ below,

$$
B_{o}=\frac{1}{2} u_{i}^{2}+\frac{p}{\rho_{o}}+\phi+\int_{0}^{t} v\left(\left(\frac{\partial u_{i}(s, \vec{x}(s))}{\partial x_{j}}\right)^{2}+\frac{\partial u_{j}(s, \vec{x}(s))}{\partial x_{i}} \frac{\partial u_{i}(s, \vec{x}(s))}{\partial x_{j}}\right) d s+I_{o}
$$

where the kinematic viscosity, $\nu$, is defined as $\frac{\mu}{\rho_{o}}$.

\section{The Laplace equation of the viscous incompressible fluid's classical Lagrangian}

The classical Lagrangian per unit mass, $L$, of an incompressible viscous fluid is defined as the kinetic energy per unit mass, $q$, minus the potential energy per unit mass, $V$. Thus, by the classical definition of the Lagrangian, $L \equiv q-V$, where the kinetic energy per unit mass, $q=u_{i}^{2}\left(t, x_{i}(t)\right) / 2$ and the potential energy per unit mass, $V=\frac{p}{\rho_{o}}+\phi$.

Theorem 1. Laplace Equation of the Fluid's Classical Lagrangian.

If the nonslip boundary condition or/and zero shear stress condition are valid at the boundaries of the viscous incompressible fluid, then the time integral of Laplacian of the classical Lagrangian of the incompressible viscous fluid equals zero for every time $t$ within the given finite time interval $[0, t 1]$.

$$
\int_{0}^{t} v \Delta L d s=0
$$

${ }^{1}$ Note $\frac{\partial B_{o}}{\partial t}$ may not be zero. 


\section{Javier Rivera}

Thus, the Laplacian of the Lagrangian function is zero, $\Delta L=0$. Or alternatively, the field derivative of the incompressible viscous Eulerian Bernoulli equation (Eq. 3) is mathematically equivalent to the addition of the dot product of the fluid velocity with the Navier-Stokes equation added to the Laplace equation of the classical Lagrangian of the incompressible viscous fluid per unit mass.

$$
\frac{d B_{o}}{d t}=u_{i}\left(\frac{\partial u_{i}}{\partial t}+u_{j} \frac{\partial u_{i}}{\partial x_{j}}+\frac{1}{\rho_{o}} \frac{\partial p}{\partial x_{i}}+\frac{\partial \phi}{\partial x_{i}}-v \Delta u_{i}\right)+v \Delta L=0
$$

Thus, the Laplace equation, $v \Delta L=0$, holds true since $\frac{d B_{0}}{d t}=0$ due to the law of total energy conservation of the fluid is constant along the stream line [1] and the NavierStokes equation is zero for viscous incompressible fluids.

Proof of Theorem 1. Laplace Equation of the Fluid's Classical Lagrangian.

The conversion of the Bernoulli equation (Eq. 3) to the integral of the Laplacian of the classical Lagrangian of the incompressible viscous fluid is pretty straight forward with the following definition of the kinetic energy per unit mass, $q$, as seen in (Eq. 4).

$$
q=u_{i}^{2}\left(t, x_{i}(t)\right) / 2
$$

The Laplacian operator, $\Delta$, applied to the kinetic energy, $q$, kinematically results in (Eq. 5).

$$
\Delta q=\left(\frac{\partial u_{i}\left(t, x_{i}(t)\right)}{\partial x_{j}}\right)^{2}+u_{i} \Delta u_{i}
$$

Or rearranging to obtain $(E q .6)$.

$$
\left(\frac{\partial u_{i}\left(t, x_{i}(t)\right)}{\partial x_{j}}\right)^{2}=\Delta q-u_{i} \Delta u_{i}
$$

Additionally, by taking the divergence of the incompressible Navier-Stokes equation is well known to result in the Laplacian of the potential energy per unit mass, $V=\frac{p}{\rho_{o}}+\phi$, as seen in (Eq. 7) [3].

$$
\frac{\partial u_{j}\left(t, x_{i}(t)\right)}{\partial x_{i}} \frac{\partial u_{i}\left(t, x_{i}(t)\right)}{\partial x_{j}}=-\Delta V
$$

By taking the field or material derivative of (Eq. 3) to obtain (Eq.8), since the total energy, $B_{o}$, is conserved along the streamline.

$$
\frac{d B_{o}}{d t}=u_{i} \frac{d u_{i}}{d t}+u_{i} \frac{\partial}{\partial x_{i}}\left(\frac{p}{\rho_{o}}+\phi\right)+v\left(\left(\frac{\partial u_{i}(s, \vec{x}(s))}{\partial x_{j}}\right)^{2}+\frac{\partial u_{j}(s, \vec{x}(s))}{\partial x_{i}} \frac{\partial u_{i}(s, \vec{x}(s))}{\partial x_{j}}\right)=0
$$

Now, inserting (Eq.6) and (Eq.7) into (Eq.8) to obtain (Eq. 9).

$$
\frac{d B_{o}}{d t}=u_{i}\left(\frac{d u_{i}}{d t}+\frac{\partial}{\partial x_{i}}\left(\frac{p}{\rho_{o}}+\phi\right)-v \Delta u_{i}\right)+v \Delta(q-V)=0
$$

Since, the Navier-Stokes equation is zero within (Eq. 9) and because the Laplacian operator is a linear operator, $(E q .9)$ can be rearrange to obtain $(E q .10)$ using the definition of the Lagrangian, $L=q-V=-\Psi$.

$$
u_{i}\left(\frac{d u_{i}}{d t}+\frac{\partial}{\partial x_{i}}\left(\frac{p}{\rho_{o}}+\phi\right)-v \Delta u_{i}\right)=-v \Delta(q-V)=-v \Delta L=v \Delta \Psi=0
$$

An alternative method is given by the use of the Navier-Stokes equation as shown in ( $E q$. 11) since kinetic viscosity $v$ is always positive scalar coefficient. Note the field or material derivative is being used in (Eq. 11).

$$
u_{i} \Delta u_{i}=\frac{u_{i}}{v}\left(\frac{d u_{i}}{d t}+\frac{1}{\rho_{o}} \frac{\partial p}{\partial x_{i}}+\frac{\partial \phi}{\partial x_{i}}\right)=\frac{1}{v} \frac{d}{d t}(q+V)
$$

Therefore, (Eq. 12) shows the equivalence of $\left(\frac{\partial u_{i}\left(t, x_{i}(t)\right)}{\partial x_{j}}\right)^{2}$ in terms of kinetic energy per unit mass, $q$, and potential energy per unit mass, $V=\frac{p\left(x_{i}(t)\right)}{\rho_{o}}+\phi\left(x_{i}(t)\right)$. 
On the Holographic Principle Description of the Viscous Incompressible Fluids

$$
\left(\frac{\partial u_{i}\left(t, x_{i}(t)\right)}{\partial x_{j}}\right)^{2}=\Delta q-\frac{1}{v} \frac{d}{d t}(q+V)
$$

Additionally, by taking the divergence of the incompressible Navier-Stokes equation is well known to result [3] in the Laplacian of the potential energy per unit mass, $V$, as seen in (Eq. 13).

$$
\frac{\partial u_{j}\left(t, x_{i}(t)\right)}{\partial x_{i}} \frac{\partial u_{i}\left(t, x_{i}(t)\right)}{\partial x_{j}}=-\Delta V
$$

Rewriting the Bernoulli equation in (Eq. 3) using (Eq. 7) and (Eq. 8) results in (Eq. 14) in terms of the potential and kinetic energy per unit mass.

$$
B_{o}=q+V+\int_{0}^{t}\left(v \Delta q-\frac{d}{d s}(q+V)-v \Delta V\right) d s+I_{o}
$$

Integrating the field derivative [1], $-\frac{d}{d s}(q+V)$, reduces (Eq. 14) to (Eq.15).

$$
B_{o}=q_{o}+V_{o}+I_{o}+\int_{0}^{t} v \Delta(q-V) d s
$$

Since $B_{o}\left(t_{0} x_{i}\left(t_{0}\right), u_{i}\left(t_{0}, x_{i}\left(t_{0}\right)\right)=B_{o}=q_{o}+V_{o}+I_{o}\right.$, then $\int_{0}^{t} v \Delta L d s=0 .{ }^{2}$ Since this equation is valid for all times within the given finite time interval $[0, t 1]$, this implies the Laplacian operator of the classical Lagrangian per unit mass, $L=q-V$, is zero.

$$
\Delta L=0
$$

Another alternate proof of the theorem can be deduced by taking the field derivative of (Eq. 14) or $(E q .15)$ to obtain $(E q .16)$ which is equivalent to $(E q .2)$ due to the incompressible Navier-Stokes equation also being obtained.

$$
\frac{d B_{o}}{d t}=v \Delta(q-V)=v \Delta L=0
$$

Thus the Laplace equation of the Lagrangian is valid for every time, $t$, within time interval $[0, t 1]$ and since the kinematic viscosity, $v$, is nonzero for viscous fluids it can be cancelled out.Thus, $(E q .2)$ is equivalent to $(E q .16)$ since the total energy of the fluid is a constant along the streamline (i.e. conserved quantity) as shown in (Eq. 17).

$$
\frac{d B_{o}(t, \vec{X}(t), \vec{u}(t, \vec{X}(t)))}{d t}=u_{i}\left(\frac{\partial u_{i}}{\partial t}+u_{j} \frac{\partial u_{i}}{\partial x_{j}}+\frac{\partial \phi}{\partial x_{i}}+\frac{1}{\rho_{o}} \frac{\partial p}{\partial x_{i}}-\frac{1}{\rho_{o}} \frac{\partial \tau_{i, j}}{\partial x_{j}}\right)=v \Delta L=0
$$

The dot or scalar product of the Navier-Stokes equation with the fluid velocity is equivalent to the Laplacian operator of the classical Lagrangian per unit mass of the viscous incompressible fluid. For ( $E q .9)$ be equivalent to (Eq. 17$)$, then the Laplacian of the Lagrangian must be identically zero. Other methods may be found to produce the same result. Notice also this result only applies for viscous flows since the kinematic viscosity needs to be positive. QED.

\section{Conclusion}

The proof of Theorem 1demonstrates that the Eulerian dynamical incompressible viscous Navier-Stokes momentum equations at a fixed time $t$ are mathematically equivalent to the Laplace equation of the classical Lagrangian of the fluid per unit mass at the same fixed time $t$. Although, a better approach is to define the Laplace equation with respect to the negative Lagrangian function, $\Psi=-L$, since $-\Delta L=\Delta(-L)=\Delta \Psi=0$ is also a Laplace equation within volume, $W_{o}$. Therefore, the Laplace equation at sample time, $t_{k}$, can be rewritten as $(E q .18)$ with Dirichlet boundary conditions, i.e. the value of the function is specified at the boundaries.

${ }^{2}$ If $f=\int_{0}^{t} v \Delta L d s=0$, then $\frac{\partial f}{\partial t}=v \Delta L=0$. 


\section{Javier Rivera}

$$
\begin{aligned}
& \Delta \Psi=0 \text { within control volume, } W_{o}, \text { at times } t_{k} \\
& \left.\Psi\right|_{\partial W_{o}}=\left.\left(\frac{p}{\rho_{o}}+\phi\right)\right|_{\partial W_{o}}, \text { at times } t_{k}
\end{aligned}
$$

The boundary conditions of $\Psi$ are due to the specified pressure and external potential, $\left(\frac{p}{\rho_{o}}+\phi\right)$, at the solid boundaries, $\partial W_{o}$, since the kinetic energy is zero due no slip boundary conditions at each sampled time $t_{k}$. In other words, $\left.\Psi\right|_{\partial W_{o}}$ is only a spatial function of the boundary for each sampled time $t_{k}$ within a finite time interval $[0, t 1]$.

If the time Fourier transforms of the fluid's velocities at Eulerian coordinate locations within the volumeare spectrally bounded by the Nyquist sampling criteria [4], then the finite time sampling of harmonic Lagrangian solutions of the Laplace equation defines a volumetric control volume $\mathrm{W}_{\mathrm{o}}$ as a "holographic frame" for each sampled time $t_{k}$ within the time interval. Therefore, the fluid's velocity flow field within the fixed control volume, $W_{o}$, may be reconstructed in time as a "holographic" Eulerian fluid flow movie with each volume frame given at the sampled times. The analogy to a hologram is due to the fact the lower dimensional boundary conditions is responsible for the Eulerian flow within the volume at a given sample time. This description is similar or analogous to the "holographic principle" described in Roger Penrose's The Road to Reality book in page 920 [2]. The solution of the Laplace equation of the classical Lagrangian per unit mass for each sample time, $t_{k}$, is a harmonic function representing Lagrangian scalar field in Eulerian coordinates, therefore, the classical Lagrangian has to be a harmonic function at each fixed sample time, $t_{k}$.

Although the fundamental solution of the Laplace equation is typically a function of the radial distance only, this might be too restrictive condition since the boundary conditions may not be radially symmetric, therefore the harmonic solutions of the Laplace equation of the classical Lagrangian per unit mass may require additional coordinates besides radial distance. The advantage of this method is that the Laplace equation is a linear equation, therefore the harmonic solutions can be superimposed to satisfy boundary conditions and the harmonic solutions of the Laplace equation are very well understood. The disadvantage of this method is that following a fluid's parcel positions will need to be clustered and tracked for different times [10], thus adding complexity to the traveling fluid parcel's position solution (so called Lagrangian coordinates of the fluid parcel).

Acknowledgements. The author would like to thank the anonymous reviewers and chief editor of APAM for their time and effort spent in reviewing this article.

\subsection{Appendix A}

The surface integral in $(E q .2 A)$ can be converted into a volume integral via Gauss divergence theorem within the arbitrary and time independent control volume $W_{o}$ :

$$
\begin{gathered}
\oiint_{\partial W_{o}} \tau_{i, j} u_{i} n_{j} d S=\iiint_{W_{o}} \frac{\partial\left(\tau_{i, j} u_{i}\right)}{\partial x_{j}} d W=0 . \\
\text { Since } \tau_{i, j} u_{i}=\mu\left(\frac{\partial u_{i}}{\partial x_{j}}+\frac{\partial u_{j}}{\partial x_{i}}\right) u_{i}=\mu\left(\frac{\partial}{\partial x_{j}}\left(\frac{1}{2} u_{i}^{2}\right)+u_{i} \frac{\partial u_{j}}{\partial x_{i}}\right) \text { and taking the divergence of } \tau_{i, j} u_{i} \text { is } \\
\frac{\partial\left(\tau_{i, j} u_{i}\right)}{\partial x_{j}}=\mu\left(\frac{\partial^{2}}{\partial x_{j}^{2}}\left(\frac{1}{2} u_{i}^{2}\right)+\frac{\partial u_{i}}{\partial x_{j}} \frac{\partial u_{j}}{\partial x_{i}}\right)
\end{gathered}
$$


On the Holographic Principle Description of the Viscous Incompressible Fluids Since $\frac{\partial u_{j}}{\partial x_{j}}=0$ by incompressibility of the fluid, $\frac{\partial^{2}}{\partial x_{j}^{2}}\left(\frac{1}{2} u_{i}^{2}\right)=\Delta q$ and $\frac{\partial u_{i}}{\partial x_{j}} \frac{\partial u_{j}}{\partial x_{i}}=-\Delta V$ therefore by substitution leads to the Laplace equation of the classical Lagrangian per unit mass, $L=q-V$.

By dividing by the constant density to obtain:

$$
\iiint_{W_{o}} \frac{\partial\left(\tau_{i, j} u_{i}\right)}{\partial x_{j}} d V=\iiint_{W_{o}} \mu \Delta q-\mu \Delta V d V=\iiint_{W_{o}} \mu \Delta L d V=0
$$

$$
\iiint_{W_{o}} \frac{1}{\rho_{o}} \frac{\partial\left(\tau_{i, j} u_{i}\right)}{\partial x_{j}} d V=\iiint_{W_{o}} v \Delta L d V=0 .
$$

Or $v \Delta L=0$ since the control volume, $W_{o}$, can be arbitrary, and is independent of time.

\section{REFERENCES}

1. J.Rivera, On field derivatives and its application to fluids, Annals of Pure and Applied Mathematics, 17(2) (2018) 163-191.

2. R.Penrose, The Road to Reality, A Complete Guide to the Laws of the Universe, Vintage Books, NY, NY, 2007, pg. 920.

3. J.Rivera, On Duhamel's solutions to the null equations of incompressible fluids, Annals of Pure and Applied Mathematics, 18(1) (2018) 169-170.

4. A.V.Oppenheim, A.S.Willsky, I.T.Young, Signals and Systems, Prentice Hall, Englewood Cliffs, New Jersey, 1983, pg. 521

5. H.Lamb, Hydrodynamics, Cambridge University Press, Cambridge, UK, (1993) pg. 46.

6. G.K.Batchelor, An Introduction to Fluid Dynamics, Cambridge Mathematical Library, Cambridge University Press, Cambridge, UK (1999) pg. 101

7. V.Jejjala, D.Minic, Y.J.Ng, C.-H.Tze, Turbulence and Holography, arXiv: 0806.0030v2 [hep-th] 30 Jun 2008, pp. 1-19

8. Z.Batiz and B.C.Chauhan, Holographic Principle and Quantum Physics, arXiv:physics/0611104v1 [physics.comp-ph] 10 Nov 2006, pp. 1-18

9. N.Banerjee and S.Dutta, Holographic hydrodynamics: models and methods, arXiv:1112.5345v1 [hep-th] 22 Dec 2011

10. J.Katz and J.Sheng, Applications of holography in fluid mechanics and particle dynamics, Annual Review of Fluid Mechanics, 42 (2010) 531-555. 